\title{
Weekly paclitaxel for a patient with advanced gastric cancer
}

\author{
Shigetaka Yamamoto ${ }^{1}$, Yasuhiro Tanaka ${ }^{1}$, Toshinori Ito ${ }^{2}$, Toyokazu Aono ${ }^{1}$, Yoshikazu Morimoto ${ }^{1}$, \\ Toru Kitagawa ${ }^{2}$, and Youjirou Kurihara ${ }^{1}$ \\ ${ }^{1}$ Department of Surgery, Osaka Prefectural General Hospital, 3-1-56 Mandaihigashi, Sumiyoshi-ku, Osaka 558-8558, Japan \\ ${ }^{2}$ Department of Surgery, Osaka University Graduate School of Medicine, Osaka, Japan
}

\begin{abstract}
A 56-year-old woman diagnosed with gastric cancer was admitted to our hospital for operation on May 15, 2001. The operation was performed on May 23. The tumor formed a large mass from the antrum to the head of the pancreas, and cancer cells were detected in the ascitic fluid microscopically. During the operation, resection was impossible, and so $100 \mathrm{mg}$ of cisplatin (CDDP) was infused into the abdominal cavity. After the operation, she experienced continuous nausea and there was a discharge of $1200-1600 \mathrm{ml}$ of digestive fluid per day from her nasogastric tube. On July 17, a new regimen, of 4-week courses of chemotherapy, with weekly administrations of $65 \mathrm{mg} / \mathrm{m}^{2}$ of paclitaxel, along with premedication for 3 weeks, followed by 1 week of rest, was started. After the first of these 4-week courses, the discharge from her nasogastric tube decreased to $200-600 \mathrm{ml}$ per day, and the tube was removed 78 days after insertion. Oral intake of food increased smoothly, and she was discharged on September 14. After another, short, hospitalization, she was discharged on October 20, and she has been coming to our outpatient clinic once a week. After paclitaxel was started, gastric fiberscopy and computed tomography (CT) scan showed reduction of the tumor. Of special note was the disappearance of ascitic fluid after two courses, rated as a "partial response" (Japanese classification). There was a decrease in hemoglobin, but neither leukocytopenia nor a decrease in platelets was found. Neuropathy was slight and no treatment was needed. Now, after 1 year, 11 courses of chemotherapy have been administered at the outpatient clinic. These results suggest weekly administration of paclitaxel to be a promising treatment for advanced gastric cancer with peritoneal dissemination. The therapeutic efficacy should be confirmed by further clinical trials.
\end{abstract}

Key words Paclitaxel $\cdot$ Advanced gastric cancer $\cdot$ Peritonitis carcinomatosa

Offprint requests to: $\mathrm{S}$. Yamamoto

Received: September 9, 2002 / Accepted: February 5, 2003

\section{Introduction}

Although successful results achieved in clinical trials of paclitaxel in patients with ovarian, breast, and lung cancer have been reported in many studies, there have been only a few published studies of such clinical trials of paclitaxel in patients with gastric cancer. At present, there is no clear consensus regarding the appropriate dosage or method of administration of paclitaxel for gastric cancer. Recently, successful results achieved using a regimen of weekly administration of paclitaxel in patients with breast cancer and ovarian cancer have been reported, but, to date, only a few such trial studies in patients with gastric cancer have been reported. Here, we report one patient in whom a regimen of weekly administration of paclitaxel appears to have been successful in treating advanced gastric cancer.

\section{Case report}

A 56-year-old woman with symptoms of heartburn and epigastralgia that had started in January 2001 was diagnosed with gastric cancer by her family doctor, and she was admitted to our hospital for operation on May 15, 2001. At that time, her abdomen was flat but slightly firm. No superficial lymph nodes were detected, and digital examination showed no signs of Schnitzler metastasis. She had slight anemia, but icterus was not detected. On hematological examination, liver and renal functions were normal, and carcinoembryonic antigen (CEA) was $1.1 \mathrm{ng} / \mathrm{ml}$. Gastric fiberscopy showed a large irregular ulcerous lesion with swelling of the folds and stiffness of the gastric wall extending from the upper dorsal to the middle body of the stomach (Fig. 1A), and this was diagnosed histologically as poorly differentiated adenocarcinoma. Abdominal computed tomography (CT) scan showed thickness of the body of the stomach, but no solitary lesions in the liver and no 


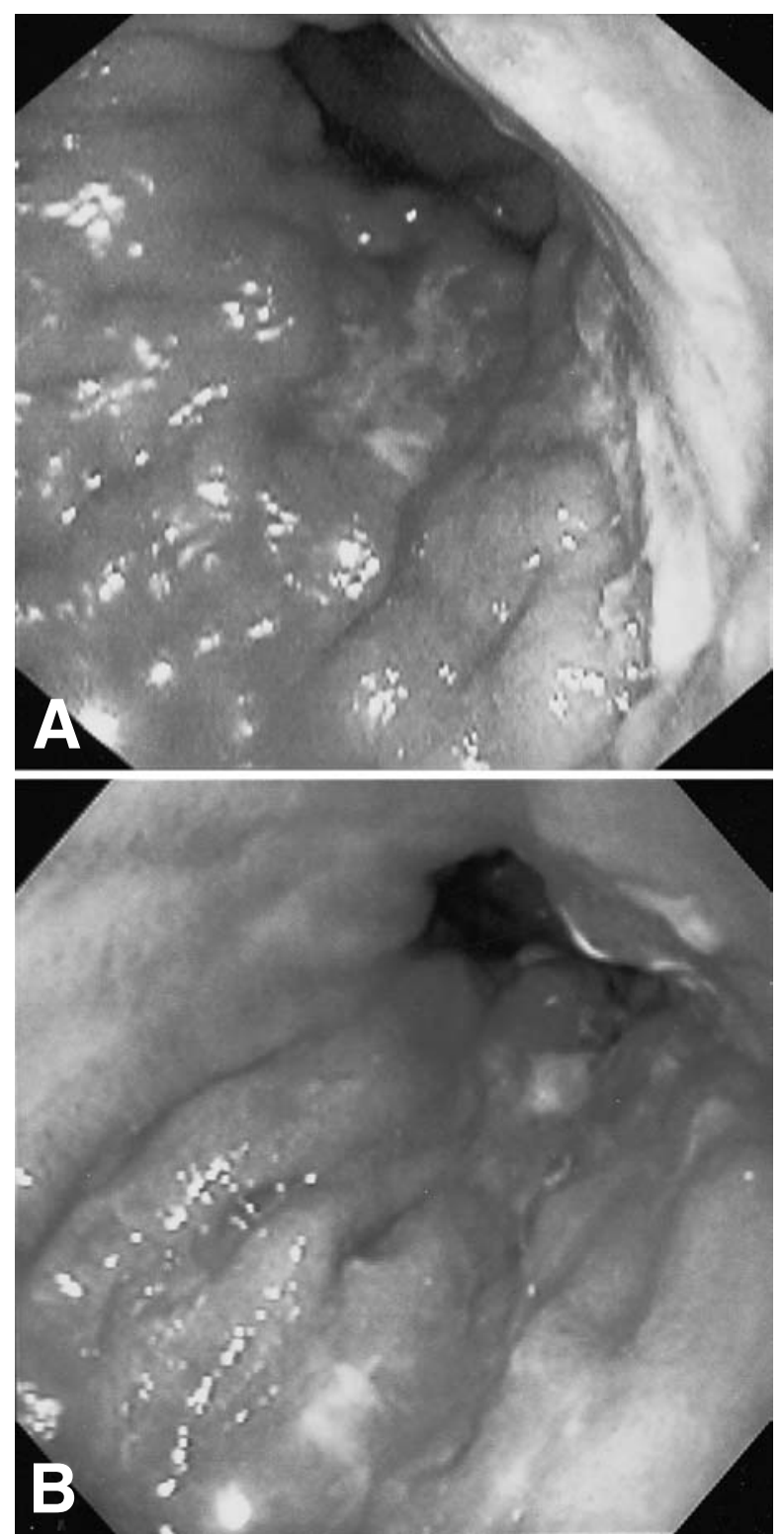

Fig. 1A,B. Gastric fiberscopy A before operation showed a large irregular ulcerous lesion with swelling of the folds and stiffness of the gastric wall, extending from the upper dorsal to the middle body of the stomach and $\mathbf{B}$ although a partial irregular ulcer still remained, the swelling of the folds and stiffness of the gastric wall were alleviated 6 months after the start of paclitaxel administration

lymph node swelling. A small amount of ascitic fluid was found in the Douglas pouch. Although peritonitis carcinomatosa was not ruled out by the results of the examinations, an operation was performed on May 23.

The tumor was found to occupy both the gastric body and the angle, and had invaded the transverse mesocolon and jejunal mesenterium near the Treitz ligament, forming a giant mass from the antrum to the head of the pancreas. Ascitic fluid was also found throughout the abdominal cavity, and cancer cells were detected in it. Neither resection nor anastomosis was able to be performed because of the extensiveness of the cancer, and so, after $100 \mathrm{mg}$ of cisplatin (CDDP) was injected into the abdominal cavity, the operation was finished.

She began eating 7 days after operation, but only small amounts, and she experienced continuous nausea. On June 5, a nasogastric tube was inserted because of vomiting. Chemotherapy, with 5-fluorouracil (5-FU) and CDDP was started on June 7, but was stopped after 1 week because the nausea became severe. Discharge of digestive fluid from the nasogastric tube was 1200$1600 \mathrm{ml}$ per day. Because of general fatigue, she remained in bed all day and her performance status was grade 3. On July 17, weekly administration of paclitaxel with a short course of premedication was started, to be given in 4-week courses, with 3 weeks of administration followed by 1 week of rest. In this regimen, $500 \mathrm{ml}$ of normal saline with vitamins was administered as a 3-h drip-infusion. After $30 \mathrm{~min}$ of infusion, $10 \mathrm{mg}$ of dexamethasone, $5 \mathrm{mg}$ of chlorpheniramine maleate, $20 \mathrm{mg}$ of famotidine, and $0.3 \mathrm{mg}$ of ramosetron hydrochloride were administered intravenously. After another $30 \mathrm{~min}$ of infusion, a dose of $65 \mathrm{mg} / \mathrm{m}^{2}$ of paclitaxel was admixed with the residual normal saline and administered for the remaining $2 \mathrm{~h}$.

After the first course of this chemotherapy regimen, discharge from her nasogastric tube decreased to 200 $600 \mathrm{ml}$ per day and urine volume increased. During the second course, the nasogastric tube was removed, 78 days after insertion. Oral intake of food increased smoothly, without nausea or vomiting, and she was discharged on September 14 (Fig. 2). Although administration of $600 \mathrm{mg} /$ day of doxifluridine had been started on September 4, it was stopped because of diarrhea, and she was readmitted to our hospital for intravenous dripinjections for 10 days. Since being discharged, she has been coming to our outpatient clinic once a week for treatment.

At 3 months after the operation, that is, 1 month after the start of paclitaxel administration, CT scan showed ascitic fluid, swelling of the gastric wall, and swelling of the greater omentum caused by remnant inflammation from peritonitis carcinomatosa (Fig. 3A). After the second course was finished though, the ascitic fluid had completely disappeared and reduction in the peritoneal lesions was found (Fig. 3B). Now, after 11 courses of chemotherapy, CT scans show no recurrence of ascitic fluid. This was rated as a "partial response" according to the Japanese classification system for gastric carcinoma [1]. At 6 months after the start of paclitaxel administration, gastric fiberscopy showed that, although a partial irregular ulcer still remained, the swelling of the folds and stiffness of the gastric wall were alleviated (Fig. 


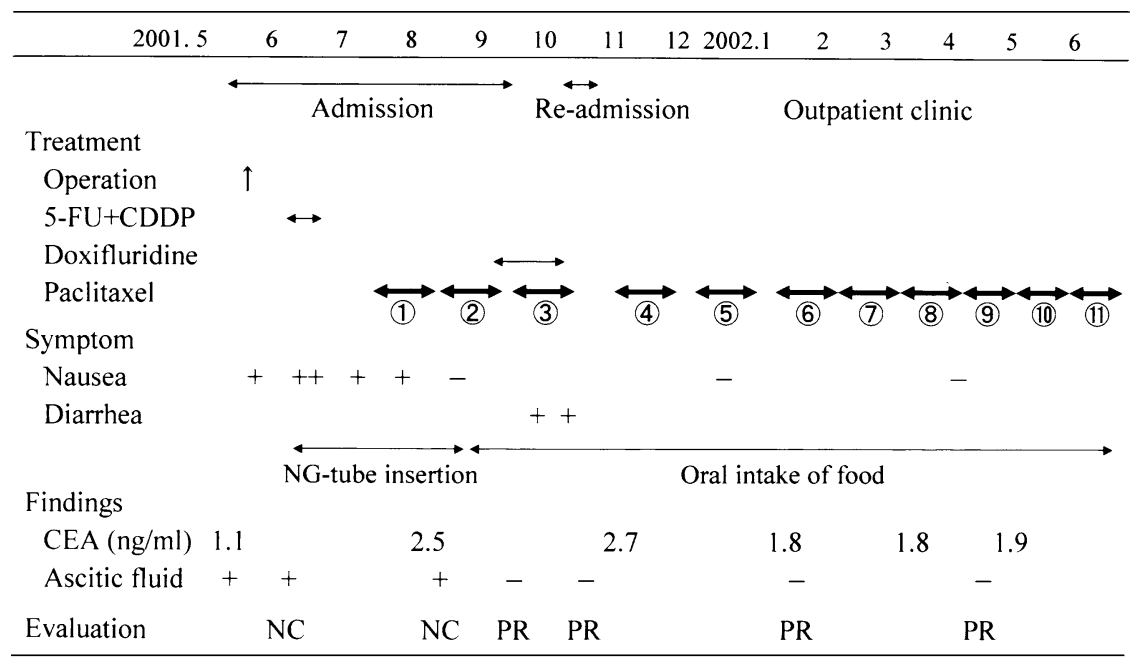

Fig. 2. Case history. 5-FU, 5-fluorouracil; $C D D P$, cisplatin; $C E A$, carcinoembryonic antigen; $N G$, nasogastric; $N C$, no change; $P R$, partial response

Table 1. Clinical trials of paclitaxel for gastric cancer

\begin{tabular}{|c|c|c|c|c|c|}
\hline Author & Dosage $\left(\mathrm{mg} / \mathrm{m}^{2}\right)$ & Interval & Combination & Rate of efficacy (\%) & MST (months) \\
\hline Cascinu et al. [5] & 225 & Every 3 weeks & None & 22 & 8 \\
\hline Ajani et al. [6] & 200 & Every 3 weeks & None & 17 & 8 \\
\hline Yamada et al. [7] & 210 & Every 3 weeks & None & 23 & 11 \\
\hline Garcia et al. [8] & 210 & Every 3 weeks & None & 11 & 5.8 \\
\hline Murad et al. [9] & 175 & Every 3 weeks & $5-\mathrm{FU}$ & 65 & 12 \\
\hline Kollmannsberger et al. [4] & 175 & Every 3 weeks & 5-FU, CDDP & 51 & 14 \\
\hline Kim et al. [10] & 175 & Every 3 weeks & 5-FU, CDDP & 51 & 6.5 \\
\hline Kuo et al. [11] & 80 & Weekly & 5-FU, leucovorin & 46 & 9 \\
\hline
\end{tabular}

MST, median survival time; 5-FU, 5-fluorouracil; CDDP, cisplatin

1B). No increase in CEA has been seen since the start of this regimen.

As for hematological side effects, although hemoglobin decreased to $7.9 \mathrm{~g} / \mathrm{dl}$ in the first course and a blood transfusion was performed, hemoglobin has remained at over $10 \mathrm{~g} / \mathrm{dl}$ since the third course. Leukocyte levels have remained at over $3000 / \mathrm{mm}^{3}$ through all courses and no decrease in platelets has been seen. While hemoglobin and leukocyte levels have tended to decrease toward the end of the third week of each course, they have repeatedly recovered during the fourth (rest) week. Increases in alanine aminotransferase and alkaliine phosphatase were also found. Some clinical symptoms, such as disturbances of taste, flushing of the face, numbness of the bottom of the feet, and pain in the ribs, along with grade 2 alopecia, were found, but they were slight and no treatment was needed.

\section{Discussion}

In recent reports of the efficacy of paclitaxel for advanced or recurrent gastric cancer, the target lesions of the chemotherapy were liver or lung metastases, gastric primary lesions, and intraabdominal lymph nodes. There are hardly any reports on the efficacy of paclitaxel for disseminated peritoneal lesions because it is difficult to evaluate efficacy in such cases. Regarding the concentration level of paclitaxel in ascitic fluid after its intravenous infusion, O'Boyle et al. [2] have reported that $0.112 \mu \mathrm{M}$ of paclitaxel was detected $7 \mathrm{~h}$ after intravenous infusion of $175 \mathrm{mg} / \mathrm{m}^{2}$ of paclitaxel, and $0.26 \mu \mathrm{M}$ of paclitaxel was detected after $22 \mathrm{~h}$, which was the same as the level in serum after $6 \mathrm{~h}$. Wiernik et al. [3] have reported that although paclitaxel was not detected in ascitic fluid during a 6-h intravenous infusion of $175 \mathrm{mg} /$ $\mathrm{m}^{2}$ of paclitaxel, it was detected subsequently, and its concentration in the ascitic fluid increased for several hours thereafter, reaching a maximum concentration of $0.25 \mu \mathrm{M}$, remaining for at least $12 \mathrm{~h}$, and then stabilizing at a level approximately $40 \%$ above that in plasma. In that same study, one patient had a massive amount of ascitic fluid due to metastatic adenocarcinoma from an unknown primary organ, yet there was only minimal sonographic evidence of ascitic fluid after 6 months of treatment with paclitaxel. Kollmannsberger et al. [4] 

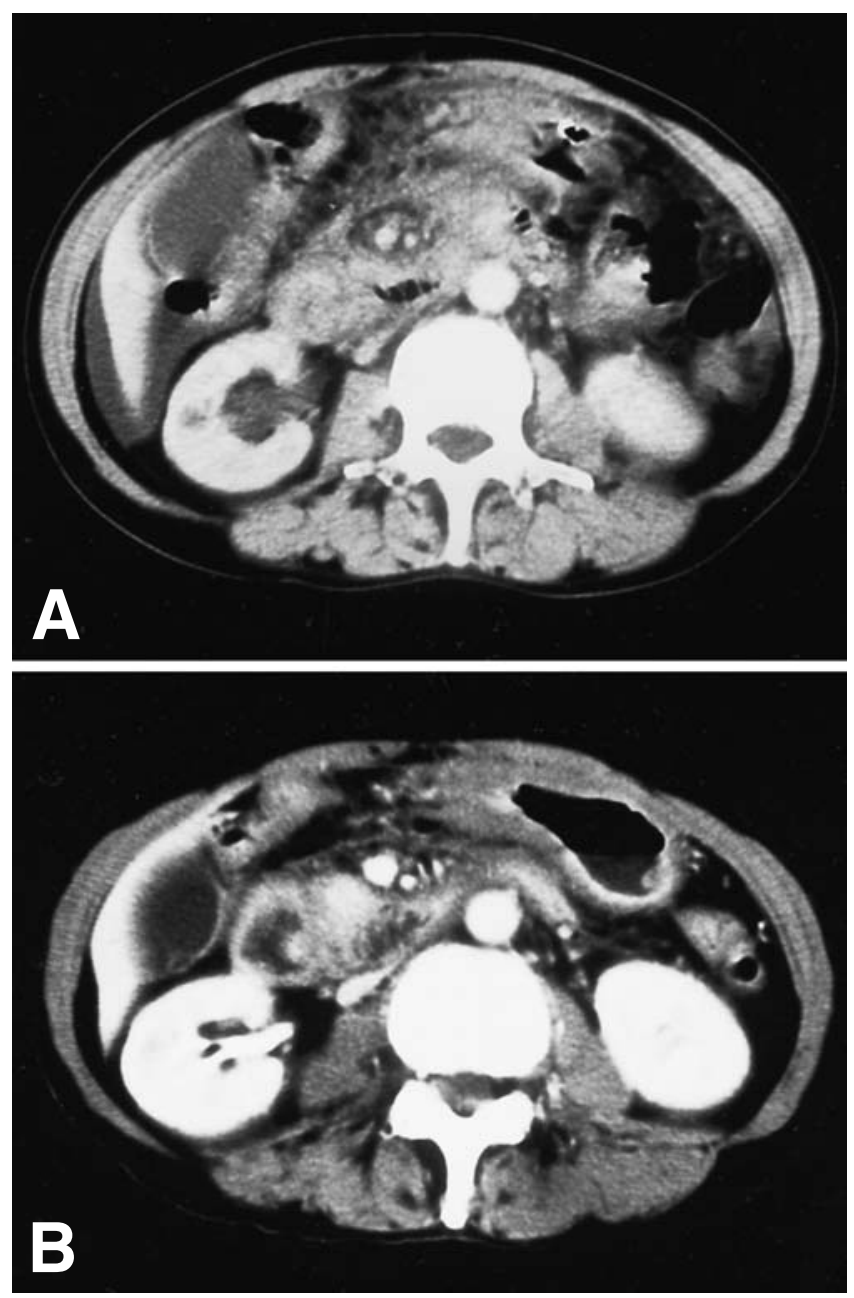

Fig. 3A,B. Computed tomography (CT) scans showed A ascitic fluid, swelling of the gastric wall, and swelling of the greater omentum 1 month after the start of paclitaxel administration; B the ascitic fluid had completely disappeared and reduction in peritoneal lesions was found after the second course was finished

have reported remission, including complete response, for 7 of 15 patients with advanced gastric cancer with peritoneal lesions, and Cascinu et al. [5] have reported an improved response for peritoneal lesions even in patients classified as stable. In our patient, the complete disappearance of the ascitic fluid and reduction of the primary gastric lesions were detected by CT scan 3 months after the start of paclitaxel. Clinical symptoms improved dramatically. Discharge volume from the nasogastric tube decreased after only one 4-week course of paclitaxel and, during the second course, the tube was able to be removed and the patient could take foods orally.

All of the clinical trials of paclitaxel for gastric cancer reported up to the present, and listed in Table 1, were performed with administration every 3 weeks [4-10], except for one [11]. With a single administration of paclitaxel every 3 weeks in one dose of $200-225 \mathrm{mg} / \mathrm{m}^{2}$, antitumor efficacy has been reported at $11 \%-23 \%$, and median survival time has been reported at 5.8-11 months. Furthermore, combination chemotherapy; for example, paclitaxel and 5-FU, or paclitaxel, 5-FU, and CDDP, has shown an improvement in these results, with antitumor efficacy at $46 \%-65 \%$ and median survival time at $6.5-14$ months. Many reports have also shown that pretreatment with other anticancer drugs had no influence on the efficacy of paclitaxel; although there was pretreatment in our chemotherapy course, our patient showed a partial response.

Some of the benefits of weekly administration of paclitaxel have already been reported, and some can be seen quite clearly in our patient. Weekly administration of paclitaxel gives no time for progression of the tumor, by shortening the intervals between drug administrations [12], and there are reports of the effectiveness of weekly administration without severe side effects in patients with breast cancer and lung cancer [13,14]. In studies where paclitaxel was administrated every 3 weeks, rates of leukopenia above grade 3 were $4.8 \%-$ $11 \%$ with paclitaxel only, and $6.8 \%-34 \%$ with combined chemotherapy [4-10]. The rate of occurrence of disturbance of nerves in the extremities over grade 1 was high, at $22 \%-75 \%$ [4-10]. In our patient, clinical symptoms, such as disturbance of taste, flushing of the face, numbness of the bottom of the feet, and pain in the ribs were seen, but no treatment was needed because of the slight degree of the symptoms. Most of these symptoms appeared after the eighth course of chemotherapy. Some hematological side effects were observed, but they disappeared after the 1 week of rest in each 4-week course.

We cannot say conclusively to what extent our results are attributable to the weekly administration of paclitaxel, or to the inclusion of a week of rest in each 4-week course, or whether our use of doxifluridine in combination with paclitaxel, albeit for a short time, may have affected the outcome. Yet, in this patient, weekly administration of paclitaxel with a 1-week rest period appears to have led to a partial response and improved performance status dramatically. Because there have been so few studies using either weekly administration of paclitaxel alone or using paclitaxel in combination with other drugs in patients with gastric cancer, we believe that this case is noteworthy and clearly points to the need for further clinical trials to confirm the therapeutic efficacy of paclitaxel in such cases. Paclitaxel could prove to be effective in the treatment of peritoneal disseminated lesions in cases of gastric cancer which previously were difficult to treat. 


\section{References}

1. Japanese Gastric Cancer Association. Japanese classification of gastric carcinoma. 2nd English Ed. Gastric Cancer 1998;1:89-100.

2. O'Boyle KP, Wang Y, Schwartz EL, Regl DE, Einzig A, Dutcher JP, et al. Development of two radioimmunoassays to detect paclitaxel in sera and in cerebrospinal, ascitic, and pleural fluids. Cancer 1997;79:1022-30.

3. Wiernik PH, Schwartz EL, Strauman JJ, Dutcher JP, Lipton RB, Paietta E. Phase I clinical and pharmacokinetic study of taxol. Cancer Res 1997;47:2486-93.

4. Kollmannsberger C, Quietzsch D, Haag C, Lingenfelser T, Schroeder M, Hartmann JT, et al. A phase II study of paclitaxel, weekly, 24-h continuous infusion 5-fluorouracil, folinic acid and cisplatin in patients with advanced gastric cancer. $\mathrm{Br} \mathrm{J}$ Cancer 2000;83:458-62.

5. Cascinu S, Graziano F, Cardarelli N, Marcellini M, Giordani P, Menichetti ET, et al. Phase II study of paclitaxel in pretreated advanced gastric cancer. Anticancer Drugs 1998;9:307-10.

6. Ajani JA, Fairweather J, Dumas P, Patt YZ, Pazdur R, Mansfield PF. Phase II study of taxol in patients with advanced gastric carcinoma. Cancer J Sci Am 1998;4:269-74.

7. Yamada Y, Shirao K, Ohtsu A, Boku N, Hyodo I, Saitoh H, et al. Phase II trial of paclitaxel by 3-h infusion for advanced gastric cancer with short premedication for prophylaxis against paclitaxel-associated hypersensitivity reactions. Ann Oncol 2001; 12:1133-7.
8. Garcia AA, Leichman CG, Lenz HJ, Baranda J, Lujan R, Casagrande Y, et al. Phase II trial of outpatient schedule of paclitaxel in patients with previously untreated metastatic, measurable adenocarcinoma of the stomach. Jpn J Clin Oncol 2001; 31:275-8.

9. Murad AM, Petroianu A, Guimaraes RC, Aragao BC, Cabral LOM, Scalabrini-Neto AO. Phase II trial of the combination of paclitaxel and 5-fluorouracil in the treatment of advanced gastric cancer. Am J Clin Oncol 1999;22:580-6.

10. Kim YH, Shin SW, Kim BS, Kim JH, Kim JG, Mok YJ, et al. Paclitaxel, 5-fluorouracil, and cisplatin combination chemotherapy for the treatment of advanced gastric carcinoma. Cancer 1999;85:295-301.

11. Kuo S, Hsu C, Yeh K, Lin J, Cheng A. A phase II study of weekly paclitaxel (taxol) and 24-h infusion of high-dose 5-fluorouracil and leucovorin (hdfl) in the treatment of advanced gastric cancer (abstract). Proc ASCO 2000;19:325.

12. Eisenhauer EA. Phase I and II trials of novel anti-cancer agents: endpoints, efficacy and existentialism. Ann Oncol 1998;9:1047-52.

13. Loffler TM, Freund W, Lipke J, Hausamen TU. Schedule- and dose-intensified paclitaxel as weekly 1 -h infusion in pretreated solid tumors: results of a phase I/II trial. Semin Oncol 1996;23 (Suppl 16):32-4.

14. Seidman AD, Hudis CA, Albanel J, Tong W, Tepler I, Currie V, et al. Dose-dense therapy with weekly 1 -h paclitaxel infusions in the treatment of metastatic breast cancer. J Clin Oncol 1998;16: 3353-61. 